\title{
Strong Orbital Polarization in a Cobaltate-Titanate Oxide Heterostructure
}

\author{
Sangjae Lee, ${ }^{1, *}$ Alex Taekyung Lee, ${ }^{2}$ Alexandru B. Georgescu, ${ }^{1,3}$ Gilberto Fabbris, ${ }^{4,5}$ Myung-Geun Han, ${ }^{4}$ \\ Yimei Zhu, ${ }^{4}$ John W. Freeland, ${ }^{5}$ Ankit S. Disa, ${ }^{2}$ Yichen Jia, ${ }^{1}$ Mark P. M. Dean, ${ }^{4}$ Frederick J. Walker, ${ }^{2}$ \\ Sohrab Ismail-Beigi, ${ }^{1,2,6}$ and Charles H. Ahn ${ }^{1,2,6}$ \\ ${ }^{1}$ Department of Physics, Yale University, New Haven, Connecticut 06520, USA \\ ${ }^{2}$ Department of Applied Physics, Yale University, New Haven, Connecticut 06520, USA \\ ${ }^{3}$ Center for Computational Quantum Physics, Flatiron Institute, New York, New York 10010, USA \\ ${ }^{4}$ Condensed Matter Physics and Materials Science Department, Brookhaven National Laboratory, Upton, New York 11973, USA \\ ${ }^{5}$ Advanced Photon Source, Argonne National Laboratory, Argonne, Illinois 60439, USA \\ ${ }^{6}$ Department of Mechanical Engineering and Materials Science, Yale University, New Haven, Connecticut 06520, USA
}

(Received 11 February 2019; revised manuscript received 21 June 2019; published 10 September 2019)

\begin{abstract}
Through a combination of experimental measurements and theoretical modeling, we describe a strongly orbital-polarized insulating ground state in an $\left(\mathrm{LaTiO}_{3}\right)_{2} /\left(\mathrm{LaCoO}_{3}\right)_{2}$ oxide heterostructure. X-ray absorption spectra and $a b$ initio calculations show that an electron is transferred from the titanate to the cobaltate layers. The charge transfer, accompanied by a large octahedral distortion, induces a substantial orbital polarization in the cobaltate layer of a size unattainable via epitaxial strain alone. The asymmetry between in-plane and out-of-plane orbital occupancies in the high-spin cobaltate layer is predicted by theory and observed through x-ray linear dichroism experiments. Manipulating orbital configurations using interfacial coupling within heterostructures promises exciting ground-state engineering for realizing new emergent electronic phases in metal oxide superlattices.
\end{abstract}

DOI: 10.1103/PhysRevLett.123.117201

Transition-metal oxides (TMOs) provide a particularly rich array of physical phenomena and phases stemming from strong electron-electron interactions between valence $d$ electrons [1-7]. In such systems where the orbital, spin, charge, and structural degrees of freedom are coupled, manipulating the orbital energies of valence electrons dramatically affects properties [8]. Orbital polarization in $3 d$ TMOs plays a crucial role in achieving high- $T_{c}$ superconductivity in cuprates [9-11], and metal-insulator transitions in the nickelates [12-14]. Understanding and controlling the electronic ground states and their orbital characteristics is crucial in realizing novel electronic properties $[15,16]$.

The perovskite cobalt oxide $\mathrm{LaCoO}_{3}$ (LCO) is of particular interest due to its versatile electronic configuration arising from interactions between the various electronic degrees of freedom. Bulk LCO has a $\mathrm{Co}^{3+}$ ground-state electronic configuration where six electrons fill the Co $3 d$ $t_{2 g}$ and $e_{g}$ shells. Because of the delicate balance among crystal field, magnetic exchange, and Hund's coupling energies, LCO has multiple competing spin states, including low-spin $\left(S=0, t_{2 g}^{6} e_{g}^{0}\right)$, intermediate-spin $\left(S=1, t_{2 g}^{5} e_{g}^{1}\right)$,

Published by the American Physical Society under the terms of the Creative Commons Attribution 4.0 International license. Further distribution of this work must maintain attribution to the author(s) and the published article's title, journal citation, and DOI. and high-spin states $\left(S=2, t_{2 g}^{4} e_{g}^{2}\right)$, whose ground-state energies differ by less than $0.1 \mathrm{eV}$ [17-19]. Traditional approaches to changing the configuration of cobalt spin states in LCO include changing temperature, pressure, and epitaxial strain $[20,21]$. For example, tensile strained LCO thin films are stabilized in the high-spin state with ferromagnetic ordering developing due to $\mathrm{CoO}_{6}$ octahedral distortions and increased $\mathrm{Co}-\mathrm{O}-\mathrm{Co}$ bond angles [22-26]. In addition, theory has elucidated a structure-property relationship where the electronic structure depends strongly on the $\mathrm{Co}-\mathrm{O}$ bond length [27]. More dramatic control of electronic structure is possible by incorporating TMOs into thin film heterostructures [28-30], exploiting quantum confinement [31] and electronic reconstructions [32]. Tuning the local structure of cobaltates and modifying the underlying energy levels allow access to different spin and orbital configurations, which can introduce various electronic and magnetic ground states.

Orbital polarization of high-spin $3 d^{7} \mathrm{Co}^{2+}$ can be achieved by inducing epitaxial strain in $\mathrm{CoO}$, but the degree of the polarization is limited by the small magnitude of strain induced by the substrate [33]. Here, we realize a strongly orbital-polarized high-spin $3 d^{7}$ state in a $\left(\mathrm{LaTiO}_{3}\right)_{2} /\left(\mathrm{LaCoO}_{3}\right)_{2}\left[(\mathrm{LTO})_{2} /(\mathrm{LCO})_{2}\right]$ heterostructure. As we show, the charge state of the cobalt is a consequence of the cross-interface charge transfer from $\mathrm{Ti}$ to $\mathrm{Co}$, and the strong orbital polarization is a consequence of the layering and structural distortions induced by the charge 
transfer [34]. Our theoretical simulation suggests that the antiferromagnetic $\mathrm{Co}$ spin configuration emerges as a result of the superexchange interaction between electrons occupying half-filled orbitals in two confined $\mathrm{CoO}_{2}$ planes. Judicious design of LCO-based heterostructures allows one to control the spin and orbital configuration of cobalt and thus manipulate the macroscopic properties through charge transfer, interfacial coupling, and dimensional confinement.

Cobaltate thin films and heterostructures were grown on $\mathrm{SrTiO}_{3}$ (STO) (001) substrates using oxygen plasma assisted molecular beam epitaxy (MBE). The layering sequence for the bilayer superlattice is $\left[(\mathrm{LTO})_{2} /(\mathrm{LCO})_{2}\right] \times 10$, and the single-component thin film consists of 40 unit cells of LCO. X-ray absorption (XAS) and x-ray linear dichroism (XLD) measurements are carried out at the beam line 4-ID-C at the Advanced Photon Source (APS). An atomic-scale structure determination is performed by measuring crystal truncation rods using high-resolution synchrotron x-ray diffraction at the beam line 33-ID-D at the APS. Real space imaging is performed using scanning transmission electron microscopy (STEM) and electron-energyloss spectroscopy (EELS). A JEOL ARM200CF with a cold field emission gun and double-spherical aberration correctors at the Brookhaven National Laboratory is operated at $200 \mathrm{keV}$. The TEM specimen is prepared using a focused ion beam (FIB) lift-out technique with $5 \mathrm{keV} \mathrm{Ga}^{+}$ion for final milling. The collection angles for high-angle annular dark-field STEM imaging are from 68 to $280 \mathrm{mrad}$. EELS data are obtained with $0.5 \mathrm{eV}$ per channel dispersion. For the theoretical calculations, we use the projector augmented wave method [35] and the revised version of the generalized gradient approximation (GGA) proposed by Perdew et al. (PBEsol, a revised Perdew-Burke-Ernzerhof GGA that improves equilibrium properties of densely packed solids and their surfaces) [36], as implemented in the Vienna ab initio simulation package software [37]. The GGA with Hubbard $U$ parameter $(\mathrm{GGA}+U)$ scheme within the rotationally invariant formalism and the fully localized limit double-counting formula is used to improve the description of electronic interactions. A plane wave basis with a kinetic energy cutoff of $500 \mathrm{eV}$ and a $9 \times 9 \times 5 \mathbf{k}$ point mesh are employed to sample the first Brillouin zone. We use $U_{\mathrm{Ti}}=3 \mathrm{eV}$ to fit the optical gap of $\mathrm{LaTiO}_{3}(0.2 \mathrm{eV})$, in line with previous work [38], and $U_{\mathrm{Co}}=2.5 \mathrm{eV}$ to describe the electronic and magnetic properties of bulk $\mathrm{LaCoO}_{3}\left(\mathrm{Co}^{3+}\right)$ and $\mathrm{CoO}\left(\mathrm{Co}^{2+}\right)$. The energy gap of LCO within GGA $+U$ using this $U_{\mathrm{Co}}$ is $0.6 \mathrm{eV}$, consistent with the experiment, and the experimental nonmagnetic insulating phase of bulk LCO is the theoretical ground state for $U_{\mathrm{Co}} \leq 2.5 \mathrm{eV}$. On the other hand, $U_{\mathrm{Co}}>4 \mathrm{eV}$ is needed to reproduce the gap of bulk $\mathrm{CoO}$. We have considered a wide range, $0 \leq U_{\mathrm{Co}} \leq 5 \mathrm{eV}$, and confirmed that our results are qualitatively unchanged for $U_{\mathrm{Co}} \geq 2 \mathrm{eV}$. (a)

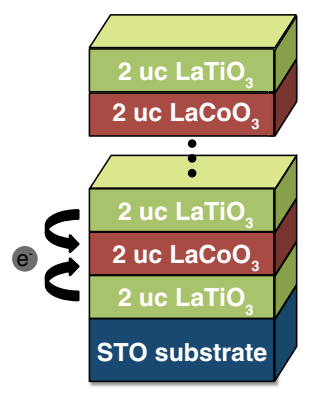

(b)

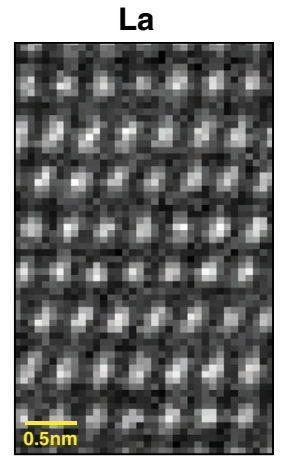

$\mathrm{Ti}$

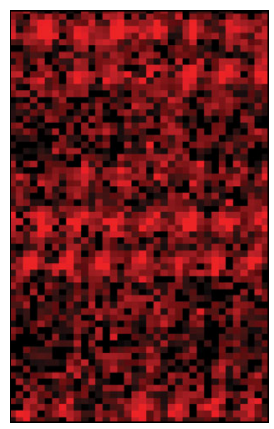

Co

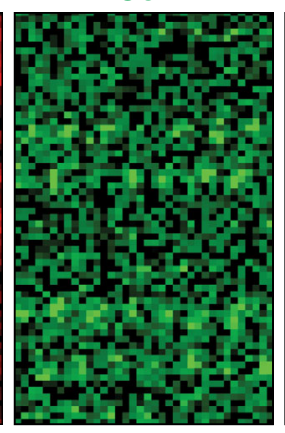

Ti+Co

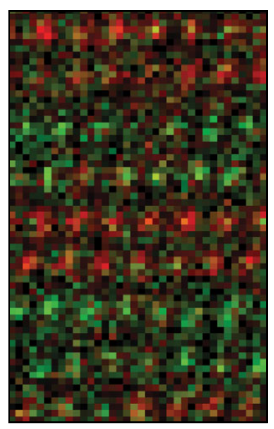

(c)

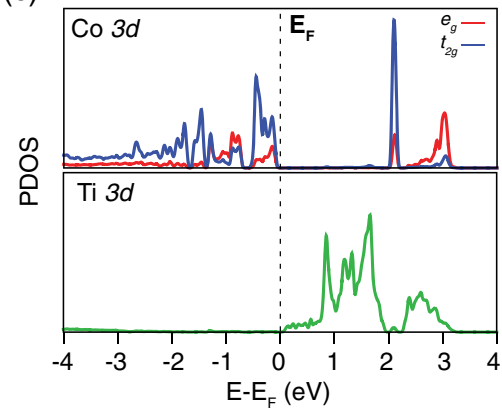

(d)

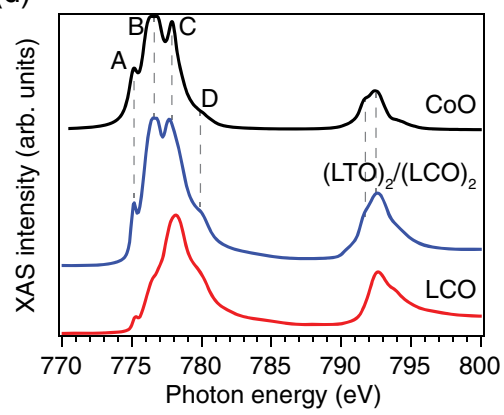

(e)

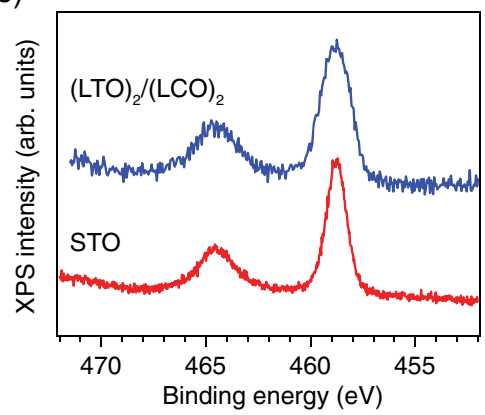

FIG. 1. (a) Schematic of the [001]-oriented (LTO $)_{2} /(\mathrm{LCO})_{2}$ heterostructure grown on top of the STO substrate. (b) Element-specific STEM-EELS maps for La, Ti, Co, and Ti + Co of the (LTO $)_{2} /(\mathrm{LCO})_{2}$ heterostructure. (c) Co and Ti $3 d$ projected density of states (PDOS) for the periodic $(\mathrm{LTO})_{2} /(\mathrm{LCO})_{2}$ superlattice from the GGA $+U$ calculations. (d) Co $L$ edge x-ray absorption spectra of the LCO thin film and the heterostructure from the experiment and the simulated absorption spectrum for bulk CoO. (e) The x-ray photoemission spectra of the Ti $2 p$ core level for the heterostructure film and the bulk STO substrate. 
The heterostructure studied here consists of two layers of LTO and two layers of LCO that form an (001)-oriented $2 / 2$ superlattice, $(\mathrm{LTO})_{2} /(\mathrm{LCO})_{2}$, illustrated in Fig. 1(a). The MBE growth process avoids intermixing of the layers to form the $\mathrm{La}_{2} \mathrm{CoTiO}_{6}$ double perovskite structure that is stable in bulk form [39-41]. The structure is characterized using STEM and EELS [Fig. 1(b) and Fig. S1 in the Supplemental Material [42]]. The element-specific STEM image shows two layers of $\mathrm{Ti}$ and $\mathrm{Co}$, consistent with limited interfacial intermixing. Larger scale characterization of the structure using X-ray diffraction (Sec. I in the Supplemental Material [42]) shows superlattice peaks arising from alternating LTO and LCO layers. The $2 / 2$ superlattice allows us to probe the interfacial phenomena effectively by having one interface per cobaltate layer, unlike heterostructures with thicker cobaltate layers, while maintaining chemical stability at the atomically abrupt interface between LTO and LCO. The difference in electronegativity $[43,44]$ between $\mathrm{Ti}^{3+}$ and $\mathrm{Co}^{3+}$ implies that the electron in the $\mathrm{Ti}^{3+} 3 d t_{2 g}$ orbital may transfer across the interface to the Co $3 d$ orbitals, modifying the oxidation states from $\mathrm{Ti}^{3+}$ to $\mathrm{Ti}^{4+}$ and from $\mathrm{Co}^{3+}$ to $\mathrm{Co}^{2+}$. This modification is confirmed by first principles calculations of the projected density of states (PDOS) for the Co and $\mathrm{Ti}$ atoms in the heterostructure, as shown in Fig. 1(c). For bulk LCO, the computed PDOS of Co $3 d$ orbitals consists of empty $e_{g}$ and fully occupied $t_{2 g}$ orbitals. In contrast, for the $(\mathrm{LTO})_{2} /(\mathrm{LCO})_{2}$ superlattice, there exist narrow $e_{g}$ bands occupied below the Fermi level, while the Ti $3 d$ bands are empty due to a full (formal) electron transfer from Ti to Co.

In order to experimentally probe this charge transfer, we measure the XAS at the Co $L$ edge which is sensitive to Co valence and spin states. The total-electron-yield absorption spectra for a LCO thin film and the $(\mathrm{LTO})_{2} /(\mathrm{LCO})_{2}$ superlattice show a significant difference [Fig. 1(d)]. We observe four peaks $A-D$ located at $775.3,776.7,777.9$, and $780.0 \mathrm{eV}$, respectively, at the Co $L_{3}$ edge XAS of the heterostructure with relative intensities $I_{A} / I_{C} \sim 0.42$, and $I_{B} / I_{C} \sim 1.01$. The spectral shape for the heterostructure resembles the experimental XAS spectra of various $\mathrm{Co}^{2+}$ compounds, including $\mathrm{CoO}$ [33] and $\mathrm{La}_{2} \mathrm{CoO}_{4}$ [45], as well as the simulated spectra for high-spin $\mathrm{Co}^{2+}$ [45-48]. To further understand the measurements, we compute the XAS spectra of bulk $\mathrm{CoO}[49,50]$, which has the same oxygen octahedral coordination as the LCO layers. The positions and relative intensities for peaks $A-D$ of the superlattice absorption spectra are reproduced well by the simulation, indicating that the heterostructure is composed mainly of the $\mathrm{Co}^{2+}$ valence state. The $\mathrm{x}$-ray photoemission spectra of the Ti $2 p$ core level are measured for the bulk STO substrate and the LTO/LCO superlattice to further illustrate the full charge transfer from $\mathrm{Ti}$ to $\mathrm{Co}$. As seen in Fig. 1(e), the heterostructure has a $\mathrm{Ti}^{4+}$ oxidation state, with $\mathrm{Ti} 2 p_{3 / 2}$ and $2 p_{1 / 2}$ peaks located at 458.8 and
$464.5 \mathrm{eV}$, respectively. Spin-orbit splitting for the superlattice is $5.71 \pm 0.10 \mathrm{eV}$, consistent with the spectra of $\mathrm{Ti}^{4+}$ in the fully oxidized STO bulk reference with spinorbit splitting of $5.60 \pm 0.10 \mathrm{eV}$. Again, the primarily $\mathrm{Ti}^{4+}$ valence state of the superlattice is consistent with crossinterface electron transfer from $\mathrm{Ti}$ to Co.

The interfacial charge transfer and resulting local electric field induce a large picoscale structural deformation in the $(\mathrm{LTO})_{2} /(\mathrm{LCO})_{2}$ superlattice. The atomic structure of the films are determined from an analysis of the measured crystal truncation rod intensities using synchrotron-based $\mathrm{x}$-ray diffraction, and the phases are derived with the coherent Bragg rod analysis (COBRA) phase retrieval technique [51,52]. A more detailed description can be found in Sec. III of the Supplemental Material [42]. The $(\mathrm{LTO})_{2} /(\mathrm{LCO})_{2}$ heterostructure [Fig. 2(b)] shows a surprisingly large oxygen displacement at the interface, where the apical oxygen at the interface is pulled towards the $\mathrm{Ti}$ atom by $\sim 0.3 \AA$, elongating the $\mathrm{CoO}_{6}$ octahedra in the outof-plane (OOP) direction. In contrast, for the LCO thin film, the measured atomic structure [Fig. 2(a)] shows no significant octahedral deformation other than the in-plane elongation of the lattice due to the $2.7 \%$ tensile strain induced by coherent epitaxy on STO (001). The ratio of the in-plane (IP) $\mathrm{Co}-\mathrm{O}$ bond length and the OOP $\mathrm{Co}-\mathrm{O}$ bond length $\left(d_{\mathrm{OOP}} / d_{\mathrm{IP}}\right)$ is 0.94 in the LCO thin film. The large oxygen displacement in the heterostructure gives $d_{\mathrm{OOP}} / d_{\mathrm{IP}}=1.14$, significantly different from the LCO thin film. These observations are very close to the computed relaxed GGA $+U$ structure [Fig. 2(c)], where an oxygen displacement is evident at the interface: the out-of-plane

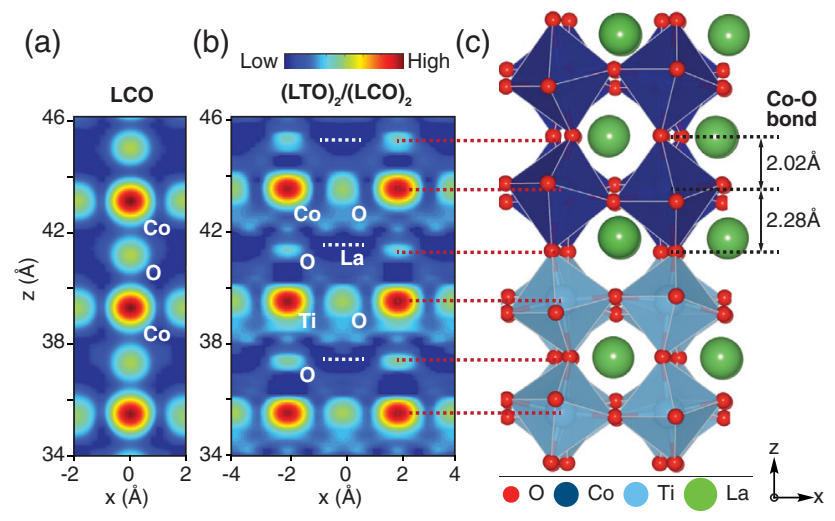

FIG. 2. A slice along a [100] plane through the $B$ site from a COBRA-derived 3D electron density map for (a) the eight unitcell thick LCO thin film and (b) a single repeat of a $(\mathrm{LTO})_{2} /(\mathrm{LCO})_{2}$ heterostructure. (c) Calculated atomic structure for the $(\mathrm{LTO})_{2} /(\mathrm{LCO})_{2}$ heterostructure from GGA $+U$. Both calculated and experimentally determined structures for the heterostructure show the large atomic displacements due to the electronic reconstruction at the cobaltate-titanate interface. La atoms are not visible in the experimental data due to the choice of the planar slice, but their $z$ coordinates as extracted from the full dataset are indicated by white dashed lines in (b). 
$\mathrm{Co}-\mathrm{O}$ bond length between the $\mathrm{CoO}_{2}$ planes is $2.02 \AA$, while the bond length between the $\mathrm{CoO}_{2}$ and $\mathrm{TiO}_{2}$ planes is $2.28 \AA$, with $d_{\mathrm{OOP}} / d_{\mathrm{IP}}=1.13$. The predicted and measured physical structure is a consequence of the interfacial charge reconstruction at the cobaltate-titanate interface that introduces an internal polar electric field pointing towards the cobalt cations, creating displacements of the apical oxygens in the $\mathrm{CoO}_{6}$ octahedra.

Theoretically, we expect that the significant elongation of the OOP Co- $\mathrm{O}$ bond will lead to an energetic splitting within both the $t_{2 g}$ and $e_{g}$ manifolds: a large orbital polarization will be induced as the on-site energy levels of the OOP $e_{g}\left(3 z^{2}-r^{2}\right), t_{2 g}(y z, z x)$ orbitals are lowered relative to the IP $e_{g}\left(x^{2}-y^{2}\right)$ and $t_{2 g}(x y)$ orbitals, respectively, changing the orbital filling of the $3 d$ orbitals. For the $\mathrm{Co}^{2+}\left(3 d^{7}\right)$ valence state in the heterostructure, we consider an additional effect where the Hubbard $U$ coupling drives the Co to adopt a high-spin configuration, where all $3 d$ spin-up orbitals are occupied, two holes occupy spin-down $e_{g}$ orbitals, and one hole occupies a spin-down $t_{2 g}$ orbital. The orbital polarization in the heterostructure puts the $t_{2 g}$ hole mainly in the $d_{x y}$ orbital [Fig. 3(c)]. The orbital polarizations of the LCO thin film and the $(\mathrm{LTO})_{2} /(\mathrm{LCO})_{2}$ superlattice are measured using soft XLD. Exploiting the symmetry of the $3 d$ orbitals and electric dipole selection rules, the occupation of IP and OOP orbitals can be probed independently by selecting the polarization parallel and perpendicular to the sample surface, respectively [53]. The absorption spectra for IP and OOP x-ray polarizations are shown in Figs. 3(a) and 3(b). The obvious dichroic (a)
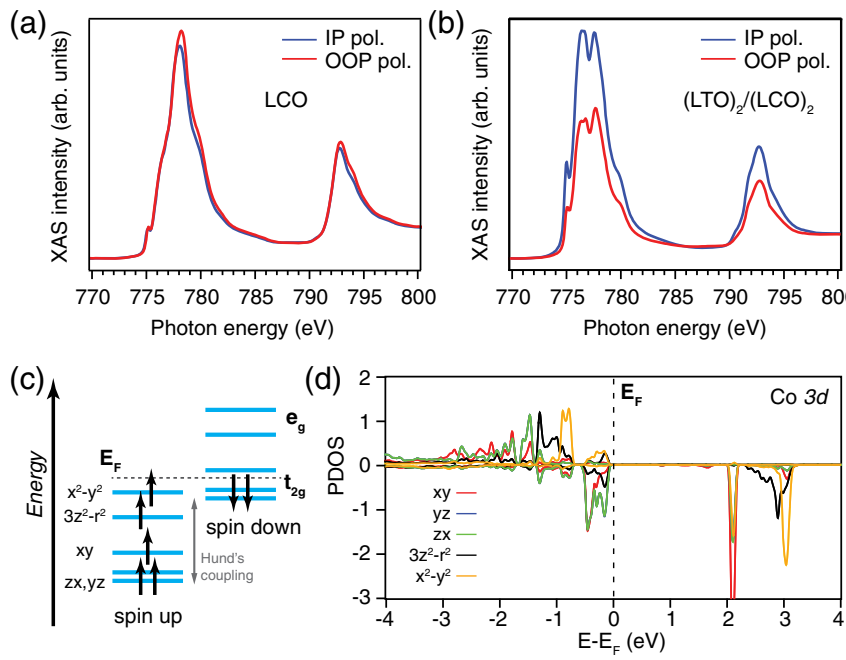

FIG. 3. Polarization-dependent x-ray absorption spectra of the (a) LCO thin film and the (b) heterostructure thin film. (c) Schematic of orbital energy levels in the $(\mathrm{LTO})_{2} /(\mathrm{LCO})_{2}$ heterostructure, which has $\mathrm{G}$ type antiferromagnetic spin ordering and a high-spin Co configuration. (d) Spin-polarized PDOS calculated for the Co $3 d$ orbitals of one Co cation in the $(\mathrm{LTO})_{2} /(\mathrm{LCO})_{2}$ superlattice (positive values for majority spin up, negative for minority spin down). The PDOS of the $y z$ orbital exactly overlaps with that of the $z x$ orbital due to symmetry. absorption signal for the heterostructure, with hole occupancy higher for the IP polarization, indicates the high-spin atomic orbital scheme shown in Fig. 3(c).

To quantify the orbital polarization, we use the OOP to IP XLD intensity ratio $r$ that describes the ratio of hole occupancies of OOP and IP orbitals in the Co $3 d$ manifold:

$r=\frac{I_{\mathrm{OOP}}}{I_{\mathrm{IP}}}=\frac{4 h_{z^{2}}+3\left(h_{y z}+h_{z x}\right)}{h_{z^{2}}+3 h_{x^{2}-y^{2}}+3 h_{x y}+1.5\left(h_{y z}+h_{z x}\right)}$,

where $h_{i}$ refers to the hole occupancy of orbital $i$. For a completely orbital-degenerate system without orbital polarization within the $t_{2 g}$ and $e_{g}$ manifolds, we have $r=1$, indicating equal hole occupancies in IP and OOP orbitals. A more detailed $a b$ initio PDOS for the $(\mathrm{LTO})_{2} /(\mathrm{LCO})_{2}$ superlattice [Fig. 3(d)] elucidates the strong energetic asymmetry of the Co $3 d$ states based on the geometry of the orbitals: spin-down $t_{2 g}$ bands of $y z, z x$ character lie below the Fermi level, while the narrow band of primarily $x y$ character [red in Fig. 3(d)] lies above the Fermi level. From the $(\mathrm{LTO})_{2} /(\mathrm{LCO})_{2}$ superlattice absorption spectra [Fig. 3(b)], we measure $r=0.70 \pm 0.03$, which is in the opposite direction of the value obtained from the LCO thin film, demonstrating the heterostructuring origin of the orbital polarization (the theoretical value is $r=0.87$ ). Note that for the fully orbital-polarized heterostructure where the spin-down $x y$ orbital is completely unoccupied, Eq. (1) yields an $r$ value of 0.57 . For the LCO thin film, we measure $r=1.06 \pm 0.03$, indicating nearly degenerate $3 d$ orbitals with a small dichroic signal due to the tensile strain from the substrate (the theoretical value is $r=1.16$; see Sec. II in the Supplemental Material for details [42]). We note that for the epitaxial LCO film, the tensile strain from the STO substrate shortens the OOP lattice constant of LCO $\left(d_{\mathrm{OOP}} / d_{\mathrm{IP}}=0.94\right)$, calculated from the atomic positions derived from the center of the electron density map in Fig. 2(a). We expect the energy level of the OOP orbitals to increase relative to the IP orbitals for the epitaxial LCO thin film, leading to $r>1$, as observed.

The consequences of this electronic structure on the spin structure can be elucidated by theory. The aligned $3 z^{2}-r^{2}$, $x^{2}-y^{2}$ orbitals of the neighboring Co atoms along the $x, y, z$ direction have a strong hybridization with the intermediate oxygen and form a strong $\mathrm{Co}-\mathrm{O}-\mathrm{Co}$ bond, giving rise to spin ordering. The superexchange interactions between the half-filled, overlapping $3 z^{2}-r^{2}, x^{2}-y^{2}$ orbitals gives a G-type antiferromagnetic spin ordering of the two cobalt layers according to our GGA $+U$ calculations. Experimentally [Fig. 4(a)], we find the absence of ferromagnetic ordering for the heterostructure, while the LCO thin film shows a ferromagnetic transition at around $77 \mathrm{~K}$. The GGA $+U$ calculation has been repeated for a variety of strains and magnetic configurations for the heterostructure, but the qualitative finding of a G-type antiferromagnetic insulating ground state with a similar electronic configuration (strong 

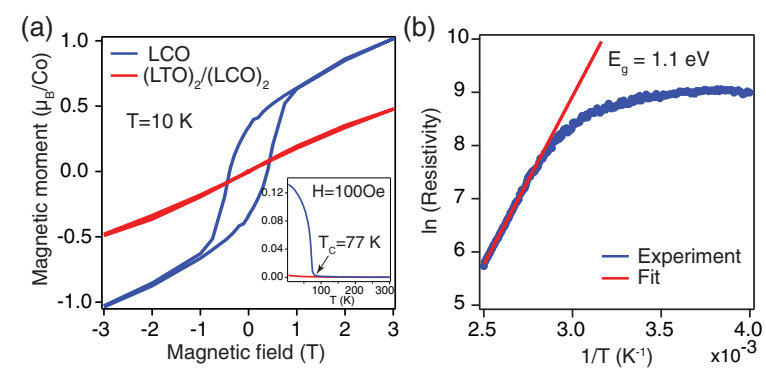

FIG. 4. (a) Magnetization vs applied magnetic field measured at $10 \mathrm{~K}$ and the magnetization vs temperature measured field cooled at $100 \mathrm{Oe}$ (inset) for the LCO thin film and the heterostructure. (b) Temperature-dependent resistivity of the $(\mathrm{LTO})_{2} /(\mathrm{LCO})_{2}$ superlattice. The red line is the linear fit of the resistivity in the high-temperature region to simple Arrhenius behavior yields the activation gap.

orbital polarization and high spin) is consistent for all strain states.

Finally, the ground state is a charge-transfer Mott-like insulator where the valence band maximum is of Co $3 d$ character, while the conduction band minimum is of $\mathrm{Ti} 3 d$ character [Fig. 1(c)]. The opening of the energy gap happens via an energetic splitting (i.e., symmetry breaking) of the energies inside the spin-down $\mathrm{Co}^{2+} t_{2 g}$ manifold: the occupied and unoccupied $t_{2 g}$ states become split by $\sim 2 \mathrm{eV}$ [Fig. 3(d)] due to the electronic interaction terms in the DFT $+U$ method. We note that breaking the crystal field symmetry via structural distortion is essential for lifting the orbital degeneracy and realizing the strong orbital polarization within the $t_{2 g}$ manifold, but it is not sufficient to realize a band gap in this high-spin system (i.e., the contribution of the $U$ term is essential). Experimentally, an insulating ground state of the heterostructure is observed in temperature-dependent electrical transport measurements. As seen in Fig. 4(b), the (LTO $)_{2} /(\mathrm{LCO})_{2}$ superlattice displays insulating behavior, with a resistivity of $\sim 300 \Omega \mathrm{cm}$, giving a sheet resistance of $\sim 100 \mathrm{M} \Omega$ at $400 \mathrm{~K}$. The high-temperature part of the figure shows that the log of resistivity is linearly proportional to the inverse of temperature, consistent with hopping transport with an activation of $E_{g} \sim 1.1 \mathrm{eV}$.

In summary, we find in this Letter a strongly orbital polarized insulating state within a $(\mathrm{LTO})_{2} /(\mathrm{LCO})_{2}$ heterostructure with antiferromagnetic spin ordering. We observe that the charge-transfer, orbital-polarization, and structural distortion patterns related to this insulating state as predicted by theory are found in experimental measurements on the heterostructure. This illustrates the possibility and feasibility of new, unique spin and orbital states in oxide heterostructures that explicitly use the motifs of orbital polarization, charge transfer, and layer confinement. Systematic study by changing the superlattice component layer thickness will enable us to modify the degree of interfacial charge transfer and to investigate its effect on the orbital polarization. Furthermore, as seen in the cuprates, this may motivate the search for a transition to correlated metallic or superconducting phases upon doping this antiferromagnetic insulator. The wide range of tunable control parameters uniquely available in perovskite heterostructures will enable us to target new electronic materials.

This work was supported by the Air Force Office of Scientific Research (AFOSR) under Grant No. FA9550-151-0472. Support for A. B. G. and S. I.-B. was provided by the National Science Foundation under Grant No. MRSEC DMR-1119826 (CRISP), and the authors acknowledge the computational support from NSF XSEDE resources through Grant No. TG-MCA08X007, the facilities and staff of the Yale Center for Research Computing, and the Scientific Computing Core division of the Flatiron Institute. The Flatiron Institute is a division of the Simons Foundation. X-ray spectroscopy measurements by G. F. and M. P. M. D. are supported by the U.S. Department of Energy, Office of Basic Energy Sciences, Early Career Award Program under Award No. 1047478. Work at Brookhaven National Laboratory was supported by the U.S. Department of Energy, Office of Science, and Office of Basic Energy Sciences, under Contract No. DESC0012704. FIB sample preparation was performed at the Center for Functional Nanomaterials, Brookhaven National Laboratory. Experiments were performed at the beam lines 4-ID-C and 33-ID-D of the Advanced Photon Source, a U.S. Department of Energy (DOE) Office of Science User Facility operated for the DOE Office of Science by Argonne National Laboratory under Contract No. DE-AC02-06CH11357.

* Corresponding author. sangjae.lee@yale.edu

[1] J. H. Ngai, F. J. Walker, and C. H. Ahn, Correlated oxide physics and electronics, Annu. Rev. Mater. Res. 44, 1 (2014).

[2] B. Lau and A.J. Millis, Theory of the Magnetic and Metal-Insulator Transitions in $\mathrm{RNiO}_{3}$ Bulk and Layered Structures, Phys. Rev. Lett. 110, 126404 (2013).

[3] A. Mogro-Campero, A review of high-temperature superconducting films on silicon, Supercond. Sci. Technol. 3, 155 (1990).

[4] C. H. Ahn, S. Gariglio, P. Paruch, T. Tybell, L. Antongnazza, and J.-M. Triscone, Electrostatic modulation of superconductivity in ultrathin $\mathrm{GdBa}_{2} \mathrm{Cu}_{3} \mathrm{O}_{7-x}$ films, Science 284, 1152 (1999).

[5] Y. Tokura and Y. Tomioka, Colossal magnetoresistive manganites, J. Magn. Magn. Mater. 200, 1 (1999).

[6] C. H. Ahn, J.-M. Triscone, N. Archibald, M. Decroux, R. H. Hammond, T. H. Geballe, Ø. Fischer, and M. R. Beasley, Ferroelectric field effect in epitaxial thin film oxide $\mathrm{SrCuO}_{2} / \mathrm{Pb}\left(\mathrm{Zr}_{0.52} \mathrm{Ti}_{0.48}\right) \mathrm{O}_{3}$ heterostructures, Science 269, 373 (1995). 
[7] C. H. Ahn, T. Tybell, L. Antognazza, K. Char, R. H. Hammond, M. R. Beasley, Ø. Fischer, and J.-M.Triscone, Local, nonvolatile electronic writing of epitaxial $\mathrm{Pb}\left(\mathrm{Zr}_{0.52} \mathrm{Ti}_{0.48}\right) \mathrm{O}_{3} / \mathrm{SrRuO}_{3}$ heterostructures, Science 276, 1100 (1997).

[8] D. Pesquera, G. Herran z, A. Barla, E. Pellegrin, F. Bondino, E. Magnano, F. Sanchez, and J. Fontcuberta, Surface symmetry-breaking and strain effects on orbital occupancy in transition metal perovskite epitaxial films, Nat. Commun. 3, 1189 (2012).

[9] L. Hozoi, L. Siurakshina, P. Fulde, and J. van den Brink, $\mathrm{Ab}$ Initio determination of $\mathrm{Cu} 3 d$ orbital energies in layered copper oxides, Sci. Rep. 1, 65 (2011).

[10] M. Moretti Sala et al., Energy and symmetry of dd excitations in undoped layered cuprates measured by $\mathrm{CuL}_{3}$ resonant inelastic x-ray scattering, New J. Phys. 13, 043026 (2011).

[11] B. Keimer, S. A. Kivelson, M. R. Norman, S. Uchida, and J. Zaanen, From quantum matter to high-temperature superconductivity in copper oxides, Nature (London) 518, 179 (2015).

[12] J. J. Peng, C. Song, M. Wang, F. Li, B. Cui, and G. Y. Wang, P. Yu, and F. Pan, Manipulating the metal-to-insulator transition of $\mathrm{NdNiO}_{3}$ films by orbital polarization, Phys. Rev. B 93, 235102 (2016).

[13] J. W. Freeland, J. Liu, M. Kareev, B. Gray, J. W. Kim, P. Ryan, R. Pentcheva, and J. Chakhalian, Orbital control in strained ultra-thin $\mathrm{LaNiO}_{3} / \mathrm{LaAlO}_{3}$ superlattices, Europhys. Lett. 96, 57004 (2011).

[14] J. Zhang, A. S. Botana, J. W. Freeland, D. Phelan, H. Zheng, V. Pardo, M. R. Norman, and J. F. Mitchell, Large orbital polarization in a metallic square-planar nickelate, Nat. Phys. 13, 864 (2017).

[15] Z. Zhong, M. Wallerberger, J. M. Tomczak, C. Taranto, N. Parragh, A. Toschi, G. Sangiovanni, and K. Held, Electronics with Correlated Oxides: $\mathrm{SrVO}_{3} / \mathrm{SrTiO}_{3}$ as a Mott Transistor, Phys. Rev. Lett. 114, 246401 (2015).

[16] Z. Liao, E. Skoropata, J. W. Freeland, E.-J. Guo, R. Desautels, X. Gao, C. Sohn, A. Rastogi, T.Z. Ward, T. Zou, T. Charlton, M. R. Fitzsimmons, and H. N. Lee, Large orbital polarization in nickelate-cuprate heterostructures by dimensional control of oxygen coordination, Nat. Commun. 10, 589 (2019).

[17] M. W. Haverkort, Z. Hu, J. C. Cezar, T. Burnus, H. Hartmann, M. Reuther, C. Zobel, T. Lorenz, A. Tanaka, N. B. Brookes, H. H. Hsieh, H. J. Lin, C. T. Chen, and L. H. Tjeng, Spin State Transition in $\mathrm{LaCoO}_{3}$ Studied Using Soft X-Ray Absorption Spectroscopy and Magnetic Circular Dichroism, Phys. Rev. Lett. 97, 176405 (2006).

[18] J. B. Goodeough, An interpretation of the magnetic properties of the perovskite-type mixed crystals $\mathrm{La}_{1-x} \mathrm{Sr}_{x} \mathrm{CoO}_{3-\lambda}$, J. Phys. Chem. Solids 6, 287 (1958).

[19] M. A. Korotin, S. Yu. Ezhov, I. V. Solovyev, V. I. Anisimov, D. I. Khomskii, and G. A. Sawatzky, Intermediate-spin state and properties of $\mathrm{LaCoO}_{3}$, Phys. Rev. B 54, 5309 (1996).

[20] P. G. Radaelli and S.-W. Cheong, Structural phenomena associated with the spin-state transition in $\mathrm{LaCoO}_{3}$, Phys. Rev. B 66, 094408 (2002).
[21] B. Rivas-Murias, I. Lucas, P. Jimenez-Cavero, C. Magen, L. Morellon, and F. Rivadulla, Independent control of the magnetization in ferromagnetic $\mathrm{La}_{2 / 3} \mathrm{Sr}_{1 / 3} \mathrm{MnO}_{3} /$ $\mathrm{SrTiO}_{3} / \mathrm{LaCoO}_{3}$ heterostructures achieved by epitaxial lattice mismatch, Nano Lett. 16, 1736 (2016).

[22] D. Fuchs, E. Arac, C. Pinta, S. Schuppler, R. Schneider, and H. v. Löhneysen, Tuning the magnetic properties of $\mathrm{LaCoO}_{3}$ thin films by epitaxial strain, Phys. Rev. B 77, 014434 (2008).

[23] A. Posadas, M. Berg, H. Seo, D. J. Smith, A. P. Kirk, D. Zhernokletov, R. M. Wallace, A. de Lozanne, and A. A. Demkov, Strain-induced ferromagnetism in $\mathrm{LaCoO}_{3}$ : Theory and growth on Si (100), Microelectron. Eng. 88, 1444 (2011).

[24] V. V. Mehta, N. Biskup, C. Jenkins, E. Arenholz, M. Varela, and Y. Suzuki, Long-range ferromagnetic order in $\mathrm{LaCoO}_{3-\delta}$ epitaxial films due to the interplay of epitaxial strain and oxygen vacancy ordering, Phys. Rev. B 91, 144418 (2015).

[25] S. Yamaguchi, Y. Okimoto, and Y. Tokura, Local lattice distortion during the spin-state transition in $\mathrm{LaCoO}_{3}$, Phys. Rev. B 55, R8666 (1997).

[26] E.-J. Guo, R. D. Desautels, D. Keavney, A. Herklotz, T. Z. Ward, and M. R. Fitzsimmons, and H. N. Lee, Switchable orbital polarization and magnetization in strained $\mathrm{LaCoO}_{3}$ films, Phys. Rev. Mater. 3, 014407 (2019).

[27] T. Ishimoto, Y. Ito, T. Tada, R. Oike, T. Nakamura, K. Amezawa, and M. Koyama, Theoretical study on temperature effect of electronic structure and spin state in $\mathrm{LaCoO}_{3}$ by using density functional theory, Solid State Ionics $\mathbf{2 8 5}$, 195 (2016).

[28] K. S. Takahashi, M. Kawasaki, and Y. Tokura, Interface ferromagnetism in oxide superlattices of $\mathrm{CaMnO}_{3} / \mathrm{CaRuO}_{3}$, Appl. Phys. Lett. 79, 1324 (2001).

[29] A. Brinkman, M. Huijben, M. van Zalk, J. Huijben, U. Zeitler, J. C. Maan, W. G. van der Wiel, G. Rijnders, D. H. Blank, and H. Hilgenkamp, Magnetic effects at the interface between non-magnetic oxides, Nat. Mater. 6, 493 (2007).

[30] A. Ohtomo and H. Y. Hwang, A high-mobility electron gas at the $\mathrm{LaAlO}_{3} / \mathrm{SrTiO}_{3}$ heterointerface, Nature (London) 427, 423 (2004).

[31] S. Stemmer and A. J. Millis, Quantum confinement in oxide quantum wells, MRS Bull. 38, 1032 (2013).

[32] A. S. Disa, D. P. Kumah, A. Malashevich, H. Chen, D. A. Arena, E. D. Specht, S. Ismail-Beigi, F. J. Walker, and C. H. Ahn, Orbital Engineering in Symmetry-Breaking Polar Heterostructures, Phys. Rev. Lett. 114, 026801 (2015).

[33] S. I. Csiszar, M. W. Haverkort, Z. Hu, A. Tanaka, H. H. Hsieh, H. J. Lin, C. T. Chen, T. Hibma, andL. H. Tjeng, Controlling Orbital Moment and Spin Orientation in CoO Layers by Strain, Phys. Rev. Lett. 95, 187205 (2005).

[34] S. Ismail-Beigi, F. J. Walker, A. S. Disa, K. M. Rabe, and C. H. Ahn, Picoscale materials engineering, Nat. Rev. Mater. 2, 17060 (2017).

[35] P. E. Blöchl, Projector augmented-wave method, Phys. Rev. B 50, 17953 (1994).

[36] J. P. Perdew, A. Ruzsinszky, G. I. Csonka, O. A. Vydrov, G. E. Scuseria, and L. A. Constantin, X. Zhou, and K. Burke, Restoring the Density-Gradient Expansion for 
Exchange in Solids and Surfaces, Phys. Rev. Lett. 100, 136406 (2008).

[37] G. Kresse and D. Joubert, From ultrasoft pseudopotentials to the projector augmented-wave method, Phys. Rev. B 59, 1758 (1999).

[38] A. T. Lee and M. J. Han, Structure, strain, and the ground state of the $\mathrm{LaTiO}_{3} / \mathrm{LaAlO}_{3}$ superlattice, Phys. Rev. B 89, 115108 (2014).

[39] A. Gómez-Pérez, M. T. Azcondo, M. Yuste, J. C. PérezFlores, N. Bonanos, F. Porcher, A. Muñoz-Noval, M. Hoelzel, F. García-Alvarado, and U. Amador, The A-cation deficient perovskite series $\mathrm{La}_{2-x} \mathrm{CoTiO}_{6-\delta}(0 \leq x \leq 0.20)$ : New components for potential SOFC composite cathodes, J. Mater. Chem. A 4, 3386 (2016).

[40] K. L. Holman, Q. Huang, T. Klimczuk, K. Trzebiatowski, J. W. G. Bos, E. Morosan, J. W. Lynn, and R. J. Cava, Synthesis and properties of the double perovskites $\mathrm{La}_{2} \mathrm{NiVO}_{6}, \mathrm{La}_{2} \mathrm{CoVO}_{6}$, and $\mathrm{La}_{2} \mathrm{CoTiO}_{6}$, J. Solid State Chem. 180, 75 (2007).

[41] M. Yuste, J. C. Pérez-Flores, J. R. de Paz, M. T. Azcondo, F. García-Alvarado, and U. Amador, New perovskite materials of the $\mathrm{La}_{2-x} \mathrm{Sr}_{x} \mathrm{CoTiO}_{6}$ series, Dalton Trans. 40, 7908 (2011).

[42] See Supplemental Material at http://link.aps.org/ supplemental/10.1103/PhysRevLett.123.117201 for details on the microscopic interfacial structure of the heterostructure.

[43] H. Chen, D. P. Kumah, A. S. Disa, F. J. Walker, C. H. Ahn, and S. Ismail-Beigi, Modifying the Electronic Orbitals of Nickelate Heterostructures via Structural Distortions, Phys. Rev. Lett. 110, 186402 (2013).

[44] H. Chen and A. Millis, Charge transfer driven emergent phenomena in oxide heterostructures, J. Phys. Condens. Matter 29, 243001 (2017).

[45] M. Merz, D. Fuchs, A. Assmann, S. Uebe, H. v. Löhneysen, P. Nagel, and S. Schuppler, Spin and orbital states in singlelayered $\mathrm{La}_{2-x} \mathrm{Ca}_{x} \mathrm{CoO}_{4}$ studied by doping- and temperaturedependent near-edge $\mathrm{x}$-ray absorption fine structure, Phys. Rev. B 84, 014436 (2011).
[46] M. Abbate, J. C. Fuggle, A. Fujimori, L. H. Tjeng, C. T. Chen, R. Potze, G. A. Sawatzky, H. Eisaki, and S. Uchida, Electronic structure and spin-state transition of $\mathrm{LaCoO}_{3}$, Phys. Rev. B 47, 16124 (1993).

[47] C. Pinta, D. Fuchs, M. Merz, M. Wissinger, E. Arac, H. v. Löhneysen, A. Samartsev, P. Nagel, and S. Schuppler, Suppression of spin-state transition in epitaxially strained $\mathrm{LaCoO}_{3}$, Phys. Rev. B 78, 174402 (2008).

[48] Z. Hu, C. Grazioli, M. Knupfer, M. S. Golden, J. Fink, P. Mahadevan, A. Kumar, S. Ray, D. D. Sarma, S. A. Warda, D. Reinen, S. Kawasaki, M. Takano, C. SchüsslerLangeheine, C. Mazumdar, and G. Kaindl, Difference in spin state, and covalence between $\mathrm{La}_{1-x} \mathrm{Sr}_{x} \mathrm{CoO}_{3}$ and $\mathrm{La}_{2-x} \mathrm{Sr}_{x} \mathrm{Li}_{0.5} \mathrm{Co}_{0.5} \mathrm{O}_{4}$, J. Alloys Compd. 343, 5 (2002).

[49] Y.L. Wang, G. Fabbris, M.P. M. Dean, and G. Kotliar, EDRIXS: An open source toolkit for simulating spectra of resonant inelastic x-ray scattering, Comput. Phys. Commun. 243, 151 (2019).

[50] M. M. Van Schooneveld, R. Kurian, A. Juhin, K. Zhou, J. Schlappa, V. N. Strocov, T. Schmitt, and F. De Groot, Electronic structure of $\mathrm{CoO}$ nanocrystals and a single crystal probed by resonant x-ray emission spectroscopy, J. Phys. Chem. C 116, 15218 (2012).

[51] Y. Yacoby, M. Sowwan, E. Stern, J. O. Cross, D. Brewe, R. Pindak, J. Pitney, E. M. Dufresne, and R. Clarke, Direct determination of epitaxial interface structure in $\mathrm{Gd}_{2} \mathrm{O}_{3}$ passivation of GaAs, Nat. Mater. 1, 99 (2002).

[52] D. P. Kumah, A. S. Disa, J.H. Ngai, H. Chen, A. Malashevich, J. W. Reiner, and S. Ismail-Beigi, F. J. Walker, and C. H. Ahn, Tuning the structure of nickelates to achieve two-dimensional electron conduction, Adv. Mater. 26, 1935 (2014).

[53] A. S. Disa, F. J. Walker, S. Ismail-Beigi, and C. H. Ahn, Research update: Orbital polarization in $\mathrm{LaNiO}_{3}$-based heterostructures, APL Mater. 3, 062303 (2015). 